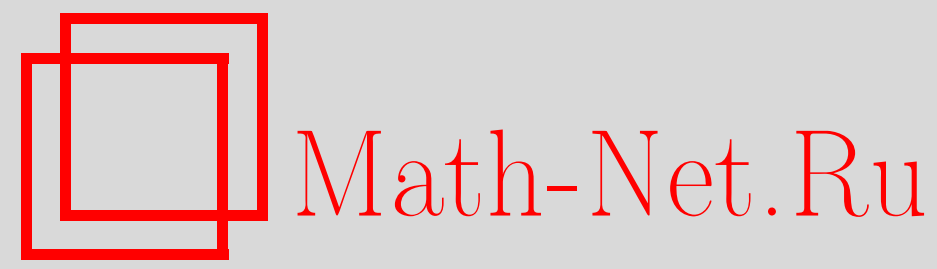

В. М. Максимов, Кубические стохастические матрицы и их вероятностные интерпретации, Теория вероятн. и ее примен., 1996, том 41, выпуск 1, 89-106

DOI: https://doi.org/10.4213/tvp2777

Использование Общероссийского математического портала Math-Net.Ru подразумевает, что вы прочитали и согласны с пользовательским соглашением http://www . mathnet.ru/rus/agreement

Параметры загрузки:

IP : 18.234 .197 .8

26 апреля 2023 г., $17: 16: 38$

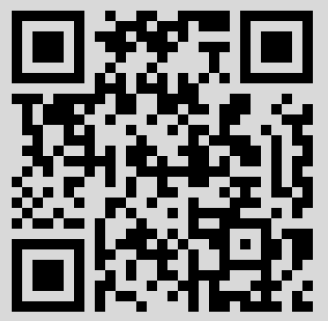




\title{
КУБИЧЕСКИЕ СТОХАСТИЧЕСКИЕ МАТРИЦЫ И ИХ ВЕРОЯТНОСТНЫЕ ИНТЕРПРЕТАЦИИ ${ }^{1)}$
}

\begin{abstract}
В работе рассматриваются ассоциативные умножения кубических матриц, обобщающие обычное матричное умножение. Вводятся кубические аналоги стохастических матриц и даются их вероятностные интерпретации. Описываются кубические стационарные стохастические матрицы и доказывается предложение о сходимости кубической стохастической матрицы к стационарной. Вводится понятие процесса марковского взаимодействия, обобшаюшее понятие марковского проџесса, и показано, что понятие эргодичности такого процесса естественно связано с ассоциативным умножением кубических матриц.
\end{abstract}

Ключевые слова и фразы: вероятность, марковская цепь, кубические стохастические матрицы, процесс марковского взаимодействия.

Введение. Обычно марковская цепь рассматривается как одна из физических интерпретаций умножения квадратных матриц. Эта интерпретация связана с так называемым каноническим базисом, который образуют канонические матрицы, обозначаемые нами $(i, j)$, $i, j=1, \ldots, s$. Матрица $(i, j)$ - это квадратная $s \times s$ матрица, единственный отличный от нуля элемент которой равен единице и стоит в $i$-й строке и в $j$-м столбце. Поскольку операпия умножения канонических матриц подчиняется правилу

$$
(i, j) \cdot(k, m)=\left\{\begin{array}{lll}
(i, m), & \text { если } & j=k, \\
0, & \text { если } & j \neq k,
\end{array}\right.
$$

где 0 обозначает нулевую $s \times s$ матрицу, то естественная вероятностная интерпретация умножения (1) приводит к марковской цепи.

Именно, символ $(i, j)$ можно интерпретировать как переход блуждающей частицы из состояния $i$ в состояние $j$ (или же, в другой интерпретации, как превращение частицы с номером $i$ в частицу с номером $j$ ). Последовательные переходы блуждающей частицы отвечают матрич-

\footnotetext{
*Тверсхой государственный университет, ул. Желябова, 33, 170000 Тверь, Росchx.

1) Работа выполнена при финансовой поддержке Российсхого фонда фундаментальных исследований (проект 93-01-1442).
} 
ному умножению (1), в то время как свойство ассоциативности этого умножения соответствует марковскому свойству блуждания.

Совокупность матриц $(i, j)$ естественным образом порождает преобразование множества $(1,2, \ldots, s)$ в себя согласно правилу $i \cdot(i, j)=j$, которое ассоциативно согласовано с умножением (1).

Ясно, что для произвольной матрищы $\left\|\alpha_{i j}\right\|$ имеет место однозначное разложение: $\left\|\alpha_{i j}\right\|=\sum \alpha_{i j} \cdot(i, j)$. Если элементы матрицы $P=\left\|p_{i, j}\right\|=$ $\sum p_{i, j}(i, j)$ удовлетворяют соотношениям $p_{i, j} \geqslant 0$ и $\sum p_{i, j}=1$, то матрица называется стохастической. Стохастическая матрица соответствует переходу блуждающей частицы из фиксированного состояния $i$ в какоелибо из состояний $(1,2, \ldots, s)$ с вероятностью 1 .

Другие базисы могут приводить к совершенно иным вероятностным интерпретациям. Рассмотрим, например, в алгебре квадратных матриц некоторую выпуклую полугруппу $\mathcal{P}$. Пусть $e_{1}, e_{2}, \ldots, e_{d}-$ ее крайние точки, т.е. такие матрицы из $\mathcal{P}$, что любая матрица $P$ из $\mathcal{P}$ единственным образом представляется в виде $P=p_{1} e_{1}+p_{2} e_{2}+\cdots+p_{d} e_{d}$, где $p_{i} \geqslant 0$ и $\sum p_{i}=1$. Тогда,

$$
e_{i} e_{j}=\sum_{k=1}^{d} p_{k}^{(i, j)} e_{k}, \quad \text { где } \quad p_{k}^{(i, j)} \geqslant 0 \quad \text { и } \quad \sum_{k=1}^{d} p_{k}^{(i, j)}=1 .
$$

Это позволяет произведение $e_{i} e_{j}$ интерпретировать как воздействие частицы $e_{i}$ на частицу $e_{j}$ (процесс взаимодействия), в результате которого с вероятностью $p_{k}^{(i, j)}$ появляется частица $e_{k}$. Поэтому степени матрицы $P^{n}=\sum_{k=1}^{d} p_{k}^{(i, j, n)} e_{k}$, точнее, вероятности $p_{k}^{(i, j, n)}, k, i, j=1, \ldots, d$, для ко̀торых $\sum_{k} p_{k}^{(i, j, n)}=1$ при любых $i, j=1, \ldots, d$ и любом натуральном $n$, можно рассматривать как результат эволющии за $n$ шагов описываемого ниже процесса.

Первоначально в некотором объеме может находиться одна из частиц типов $e_{1}, \ldots, e_{d}$ с вероятностями $p_{1}, \ldots, p_{d}$ соответственно. По находящейся в этом объеме с вероятностью $p_{j}$ частице $e_{j}$ мы в первый момент времени с вероятностью $p_{i}$ выстреливаем частицей $e_{i} . \mathrm{B}$ peзультате в тот же момент с вероятностью $p_{i} p_{j} p_{k}^{(i, j)}$ появляется частица $e_{k}$. После этого процесс выстреливания продолжается во второй момент времени, третий и т.д. Ясно, что вероятность $p_{k}^{(i, j, n)}, k=1, \ldots, d$, есть вероятность появления частицы $e_{k}$ после $n$-го выстрела.

Можно было бы рассмотреть и другие физические интерпретации вероятностей $p_{k}^{(i, j, n)}$, например, на языке химической кинетики, катализа или даже демографик. Однако, с математической точки зрения это не имеет особого значения. Важно другое. В возникшем в результате интерпретации матричной выпуклой полугрупшы $\mathcal{P}$ процессе взаимодействия можно так изменить параметры взаимодействия, что процесс уже не будет управляться произведением квадратных матриц из $\mathcal{P}$. В качестве примера можно предложить ситуацию, когда выстреленные с 
вероятностью $p_{i}$ частищы $e_{i}$ попадают в частищу $e_{j}$ с вероятностью $p_{i, j}$, $\sum_{i, j} p_{i, j}=1$, и в результате их взаимодействия появляется частица $e_{k}$ с вероятностью $p_{i, j, k}, \sum_{k} p_{i, j, k}=1$. Возникающий таким образом дискретный по времени процесс мы будем называть процессом марковского взаимодействия, и он будет рассмотрен ниже. Разница в описании процессов принципиальна. В первом случае элементы кубической матрицы $\left\|p_{k}^{(i, j)}\right\|$ являются структурными константами матричной выпуклой полугруппы и поэтому не могут быть произвольными. Во втором случае матрица $\left\|p_{i, j, k}\right\|$ фактически произвольна: на нее наложены лишь условия стохастичности $p_{i, j, k} \geqslant 0, \sum_{k} p_{i, j, k}=1$. Кроме того, в образовании процесса участвует произвольная квадратная стохастическая матрица $\left\|p_{i, j}\right\|$.

Представляет интерес слепуюший вопрос: какие интерпретации в терминах блужданий частиц или их взаимодействий можно найти для многоразрядных матриц, если подходящим способом определить для них операцию умножения, обладаюшую свойством ассоциативности?

Поскольку при рассмотрении многоразрядных (многомерных, или пространственных) матриц число нетривиальных ассоциативных операций естественно возрастает, то можно ожидать, что им отвечают новые понятия многомерных стохастических матриц и соответствуюшие им вероятностные интерпретации.

В настоящей работе на примере кубических матриц приводится одна из реализаций этой идеи. С многомерными матрицами и их приложениями можно познакомиться в [1].

1. Ассоциативные умножения кубических матриц. Пусть $\Gamma$ - произвольный конечный алфавит из $s$ элементов, т.е. конечное множество без каких бы то ни было дополнительных структур на нем. Аналогично квадратным каноническим матрицам, определим канонические тройки $(i, j, k), i, j, k \in \Gamma$, которые будут образовывать кубический канонический базис, относительно которого каждая кубическая матрица $\left\|\alpha_{i, j, k}\right\|, i, j, k \in \Gamma$, однозначно представляется в виде $\left\|\alpha_{i, j, k}\right\|=$ $\sum_{i, j, k} \alpha_{i, j, k} \cdot(i, j, k)$.

В следующих ниже рассмотрениях конечность множества $\Gamma$ фактически не имеет значения, если рассматривать кубические матрицы, допускаюшие лишь конечное разложение по каноническому базису.

По аналогии с умножением (1) определим для троек $(i, j, k)$ элементов из Г следующие умножения:

$$
\begin{aligned}
& \text { I. }(i, j, k) \cdot(m, n, r)= \begin{cases}(i, j, r), & \text { если } k=m \text { и } j=n, \\
0 & \text { в противном случае; }\end{cases} \\
& \text { II. }(i, j, k) \cdot(m, n, r)= \begin{cases}(i, j, r), & \text { если } k=m, \\
0 & \text { в противном случае; }\end{cases} \\
& \text { III. }(i, j, k) \cdot(m, n, r)= \begin{cases}(i, n, r), & \text { если } k=m, \\
0 & \text { в противном случае. }\end{cases}
\end{aligned}
$$


Ассоциативность введенных умножений легко проверяется. Проще всего устроено умножение (2), сводящееся к обычному матричному умножению. Действительно, справедливо

Предложение 1. Алгебра кубических матрич с умножением (2) является прямой суммой алгебр квадраттных матрич.

Д о к а 3 а т е л ь с т в о. Ясно, что совокупность канонических матриц $(i, j, k)$ при фиксированном $j$, ввиду определения (2), является полугруппой, которая при отображении $(i, j, k) \longrightarrow(i, k)$ изоморфна полугруппе, образованной каноническим базисом $(i, k)$ с умножением (1). Следовательно, $j$-е сечение в алгебре матриц с умножением (2) изоморфно алгебре квадратных матриц с умножением (1). С другой стороны, снова ввиду определения (2), произведения различных сечений равны нулю. Предложение доказано.

Умножения (3) и (4) являются частными случаями более общей операции. В самом деле, если на алфавите $Г$ задана произвольная ассоциативная операция о, то для канонических матриц $(i, j, k)$ можно определить умножение

IV. $(i, j, k) \cdot(l, m, r)= \begin{cases}(i, j \circ m, r), & \text { если } k=l, \\ 0 & \text { в противном случае. }\end{cases}$

Ассоциативность умножения (5) вытекает из следующей цепочки очевидных равенств:

$$
\begin{aligned}
& {[(i, j, k) \cdot(k, n, r)] \cdot(r, s, t)=(i, j \circ n, r) \cdot(r, s, t)=(i,(j \circ n) \circ s, t)} \\
& (i, j, k) \cdot[(k, n, r) \cdot(r, s, t)]=(i, j, k) \cdot(k, n \circ s, t)=(i, j \circ(n \circ s), t) .
\end{aligned}
$$

Если операцию о на Г определить соотношением $j \circ n=j$ (ассоциативность этой операции очевидна), то умножение (5) превращается в (3). Аналогично, ассоциативная операция $j \circ n=n$ на $\Gamma$ превращает (5) в (4).

Отметим, что для первой из рассмотренных отераций уравнение $x \circ a=b$, а для второй - уравнение $a \circ x=b$ однозначно разрешимы при любых $a, b \in \Gamma$.

В дальнейшем всегда будем предполагать, что операция о на Г обладает либо правой, либо левой однозначной разрешимостью. Справедлива следующая

Лемма 1. Если операция о на Г обладает либо правой, либо левой однозначной разрешимостью, то сумма $\sum_{d \in \Gamma} \sum_{j \circ m=d} \gamma_{j, m}$ совпадает с суммой $\sum_{j \in \Gamma} \sum_{m \in \Gamma} \gamma_{j, m}$.

Д о к а з а т е л ь с т в о. Пусть, например, операция о обладает левой однозначной разрешимостью. Тогда для любых $m$ и $d \in \Gamma$ однозначно определен элемент $j_{d}$ как решение уравнения $j_{d} \circ m=d$, причем при разных $d$ элементы $j_{d}$ различны. Поэтому множество $\left\{j_{d}, d \in \Gamma\right\}$ для любого фиксированного $m \in \Gamma$ есть перестановка множества $\Gamma$. Следо- 
вательно,

$$
\sum_{d \in \Gamma} \sum_{j \circ m=d} \gamma_{j, m}=\sum_{m \in \Gamma} \sum_{j_{d} \circ m, d \in \Gamma} \gamma_{j, m}=\sum_{m \in \Gamma} \sum_{j \circ m, j \in \Gamma} \gamma_{j, m}=\sum_{m \in \Gamma} \sum_{j \in \Gamma} \gamma_{j, m} .
$$

Лемма доказана.

Предложение 2. Алгебра кубических матрич с операчией (5) изоморфна тензорному произведению алгебры квадратных матрич и полугрупповой алгебры с базисом $\Gamma$ и операцией $о$.

Д ок а з а т е л с т в о. Из определения (5) следует, что взаимно однозначное сопоставление базисной тройке $(i, j, k)$ элемента $(i, j) \otimes k$ из тензорного произведения задает гомоморфизм. Так как тройки линейно независимы в алгебре кубических матриц, а элементы $(i, j) \otimes k$ образуют базис тензорного произведения, то, продолжая гомоморфизм по линейности, получаем требуемый изоморфизм.

2. Стохастические кубические матрицы. Из предложения 2 ясно, что прямое произведение квадратной стохастической матрицы $\left\|p_{i, j}\right\|$ и распределения $\mu=\left\{p_{k}\right\}$ на $\Gamma$ задает кубическую матрицу $P=$ $\left\|p_{i, j, k}\right\|$, где $p_{i, j, k}=p_{i, j} p_{k}$, причем, очевидно,

$$
\sum_{j, k} p_{i, j, k}=\sum_{k} p_{k} \sum_{j} p_{i, j}=\sum_{k} p_{k}=1
$$

Если аналогично определить кубическую матрицу $Q=\left\|q_{r, s, t}\right\|$, где $q_{r, s, t}=q_{r, s} q_{t},\left\|q_{r, s}\right\|$ - стохастическая матрига, а $\nu=\left\{q_{t}\right\}-$ pacmpeделение на $\Gamma$, то ясно, что кубическая матрица $P Q$ является прямым произведением матрицы $\left\|p_{i, j}\right\| \cdot\left\|q_{r, s}\right\|$ и распределения $\mu \circ \nu$ на $Г$. Другими словами, если рассматривать введенное прямое произведение как трехмерный аналог стохастических квадратных матрищ, то так определенная стохастичность сохраняется при умножении. К сожалению, такое множество стохастических матриц недостаточно в приложениях. Очевидно, однако, что всякое его расширение должно сохранять свойство (6). Поэтому естественно ввести

О п р е д е л е н и е 1 . Кубическая матрица $P=\left\|p_{i, j, k}\right\|$ называется стохастической, если $p_{i, j, k} \geqslant 0$ и $\sum_{j, k \in \Gamma} p_{i, j, k}=1$ для всех $i \in \Gamma$.

В дальнейшем, если специально не оговорено, все утверждения рассматриваются для алтебры кубических матриц с операцией (5).

Предложение 3. Стохастические кубические матриць образуют выпуклую полугрупnу.

Д ок а за т е л ь с т в о. Если $P=\left\|p_{i, j, k}\right\|$ и $Q=\left\|q_{r, s, t}\right\|$ - две стохастические кубические матрицы, то для $(i, j \circ m, r)$-го элемента $t_{i, j \circ m, r}$ произведения $P Q$, согласно определению (5), имеем:

$$
t_{i, d, r}=\sum_{j, m} \sum_{k} p_{i, j, k} q_{k, m, r}, \quad j \circ m=d .
$$


Поэтому, используя лемму 1 и определение 1 , получим

$$
\begin{aligned}
\sum_{d, r} t_{i, d, r} & =\sum_{r} \sum_{d=j \circ m} \sum_{k} p_{i, j, k} q_{k, m, r}=\sum_{k} \sum_{r} \sum_{j} \sum_{m} p_{i, j, k} q_{k, m, r} \\
& =\sum_{k, j} p_{i, j, k} \sum_{m, r} q_{k, m, r}=1 .
\end{aligned}
$$

Свойство выпуклости рассматриваемой совокупности кубических матриц очевидно. Предложение доказано.

Пусть теперь $\left\|p_{i, j}\right\|$ - дважды стохастическая квадратная матрица и $\mu=\left\{p_{k}\right\}$ - распределение на Г. В этой ситуации для кубической матрицы $P=\left\|p_{i, j, k}\right\|$ с элементами $p_{i, j, k}=p_{i, j} p_{k}$ имеем: $\sum_{i} p_{i, j, k}=$ $p_{k} \sum_{i} p_{i, j}=p_{k}$. Сумма $\sum_{i} p_{i, j, k}$ зависит только от $k$. Если же все $p_{k}$ равны между собой, т.е. равны $1 / s$ ( $s$ - размерность матрицы), то сумма не зависит ни от $j$, ни от $k$. Этим оправдано

О п р е д е л е п и е 2. Стохастическая кубическая матрица называется дважды стохастической, если $\sum_{i} p_{i, j, k}=1 / s$ при всех $j, k \in \Gamma$.

Предложение 4. Дважды стохастические кубические матрицы образуют выпуклую полугруппу.

Д о к а 3 а т е л ь с т в о. В обозначениях, использованных при доказательстве предложения 3 , имеем:

$$
\begin{aligned}
\sum_{i} t_{i, d, r} & =\sum_{i} \sum_{m, j \in \Gamma ; j \circ m=d} \sum_{k} p_{i, j, k} q_{k, m, r}=\sum_{j, m ; j \circ m=d} \frac{1}{s} \sum_{k} q_{k, m, r} \\
& =\frac{1}{s^{2}} \sum_{j, m ; j \circ m=d} 1=\frac{s}{s^{2}}=\frac{1}{s},
\end{aligned}
$$

ибо сумма взята по $j$ и $m$ таким, что $j \circ m=d$, и при любом фиксированном $d \in \Gamma$ имеет, в силу свойства однозначной (правой или левой) разрешимости, ровно $s$ членов. Свойство линейной выпуклости очевидно.

Понятие дважды стохастичности можно существенно расширить при увеличении размера матрицы.

$\mathrm{O}$ п р е д е ле н и е 3 . Кубическая матрица $P=\left\|p_{i, j, k}\right\|$ с неотрицательными элементами называется дважды стохастической по первому, второму или третьему индексу, если выполнено, соответственно, первое, второе или третье из равенств

$$
\begin{array}{lll}
\sum_{i} p_{i, j, k}=\frac{1}{s} & \text { при любых } & j, k \in \Gamma ; \\
\sum_{j} p_{i, j, k}=\frac{1}{s} & \text { при любых } & i, k \in \Gamma ; \\
\sum_{k} p_{i, j, k}=\frac{1}{s} & \text { при любых } & i, j \in \Gamma .
\end{array}
$$


Предложение 5. Множество кубических матрич, дважды стохастических относительно или первого, или второго, или же третьего индекса, образуют выпуклую полугруппу.

Д о к а 3 а т е л в с т в о. Прежде всего заметим, что то, что в определении 2 мы называем дважды стохастичностью, есть дважды стохастичность по первому индексу. Поэтому предложение достаточно доказать для дважды стохастичности относительно второго и третьего индексов.

Выпуклость рассматриваемых совокупностей очевидна. Проверим полугрупповое свойство для матриц, дважды стохастических относительно второго индекса. Используя обозначения, принятые в доказательстве предложения 4 , имеем:

$$
\begin{aligned}
\sum_{d} t_{i, d, r} & =\sum_{d} \sum_{j \circ m=d} \sum_{k} p_{i, j, k} q_{k, m, r} \\
& =\sum_{k} \sum_{j} \sum_{m} p_{i, j, k} q_{k, m, r}=\sum_{k} \frac{1}{s^{2}}=\frac{s}{s^{2}}=\frac{1}{s} .
\end{aligned}
$$

Доказательство для третьего индекса аналогично.

Заметим, что дважды стохастичность матрицы $P=\left\|p_{i, j, k}\right\|$ по второму или третьему индексу влечет стохастичность $P$. В самом деле, $\sum_{j} p_{i, j, k}=1 / s$. Тогда, очевидно,

$$
\sum_{j, k} p_{i, j, k}=\sum_{k} \sum_{j} p_{i, j, k}=\frac{1}{s} \sum_{k} 1=\frac{s}{s}=1 .
$$

Это приводит к мысли, что стохастичность кубической матрицы может быть определена для любой пары ее индексов. В исходном определении 1 стохастическую матрицу естественно называть стохастической типа $(2,3)$. Аналогично, назовем кубическую матрищу $P=\left\|p_{i, j, k}\right\|$ стохастической типа $(1,2)$ (соответственно, типа $(1,3)$ ), если $\sum_{i, j} p_{i, j, k}=1$ при всех $k \in \Gamma$ (соответственно, $\sum_{i, k} p_{i, j, k}=1$ для всех $j \in \Gamma$ ).

Предложение 6. Совокупности стохастических матрич типа $(1,2)$ и типа $(2,3)$ образуют выпуклые полуаруппы.

Д о к а з а т е л ь с т в о достаточно провести, в силу предложения 3 , для стохастических матриц типа $(1,2)$. В обозначениях, использованных при доказательстве предложения 3 , имеем:

$$
\sum_{i, d} t_{i, d, r}=\sum_{i} \sum_{d} \sum_{j \circ m=d} \sum_{k} p_{i, j, k} q_{k, m, r}=\sum_{m}\left(\sum_{k} \sum_{i, j} p_{i, j, k}\right) q_{k, m, r}=1 .
$$

Предложение доказано.

3 а м е ч а н и е. Любопытно отметить, что совокупность стохастических матриц типа $(1,3)$ не образует полугруппу относительно умножения. Более того, легко построить примеры, когда произведение стохастических матриц типа $(1,3)$ равно нулю. С этой целью представим $\Gamma$ 
как сумму непересекаюшихся множеств $\Gamma_{1}$ и $\Gamma_{2}$. Определим $\mathcal{P}_{\Gamma_{1}}, \Gamma_{2}$ как совокупность матриц $P=\left\|p_{i, j, k}\right\|$ таких, что $p_{i, j, k}=0$, если либо $i \notin \Gamma_{1}$, либо $k \notin \Gamma_{2}$. Тогда, если матрицы $P=\left\|p_{i, j, k}\right\|$ и $Q=\left\|q_{r, s, t}\right\|$ принадлежат $\mathcal{P}_{\Gamma_{1}, \Gamma_{2}}$, то $\sum_{m} \sum_{k} p_{i, j, k} q_{k, m, r}=0$ при любых $i, j, r$, т.е. $P Q=0$. С другой стороны, если $i \in \Gamma_{1}$ и $k \in \Gamma_{2}$, то при любом фиксированном $j$ найдутся положительные элементы $p_{i, j, k}$, дающие в сумме 1.

Приведенная конструкция неприменима в случае стохастических матриц типов $(1,2)$ или $(2,3)$, поскольку, например, при $k \notin \Gamma_{2}$ все $p_{i, j, k}=0$, и поэтому $\sum_{i, j} p_{i, j, k}=0$.

Пусть $\varphi$ - отображение, ставящее кубической матрище $\left\|p_{i, j, k}\right\|$ в соответствие квадратную матрицу $\left\|\tilde{p}_{i, k}\right\|$, гце $\tilde{p}_{i, k}=\sum_{j} p_{i, j, k}$. Матрицу $\left\|\widetilde{p}_{i, k}\right\|$ мы будем называть сопровождающей матрицей матрицы $\left\|p_{i, j, k}\right\|$. Если $P$ - стохастическая матрица типа $(2,3)$, то ее сопровождающая матрица, очевидно, есть обычная стохастическая матрица.

Предложение 7. Отображение $\varphi$ гомоморфно отображает алгебру кубических матрич с операцией умножения (5) в алгебру квадратных матрич с обычным умножением.

Доказат т ль с т в о. Пусть $P=\left\|p_{i, j, k}\right\|$ и $Q=\left\|q_{r, s, t}\right\|-$ произвольные кубические матрищы. Элемент произведения $P Q$, стояший на $(i, d, r)$-м месте, есть $t_{i, d, r}=\sum_{j, m} \sum_{k} p_{i, j, k} q_{k, m, r}, j \circ m=d$. Поэтому при отображении $\varphi$ получим $\tilde{t}_{i, r}=\sum_{d} \sum_{j, m} \sum_{k} p_{i, j, k} q_{k, m, r}, j \circ m=d$, и, воспользовавшись леммой 1 , найдем, что

$$
\tilde{t}_{i, r}=\sum_{j} \sum_{m} \sum_{k} p_{i, j, k} q_{k, m, r}=\sum_{k}\left(\sum_{j} p_{i, j, k}\right)\left(\sum_{m} q_{k, m, r}\right)=\sum_{k} \tilde{p}_{i, k} \widetilde{q}_{k, r} .
$$

Линейность же отображения $\varphi$ очевидна. Предложение доказано.

В заключение этого пункта введем стохастические кубические матрицы относительно операции умножения (2). Приходится это делать отдельно, поскольку, в силу предложений 1 и 2 , это умножение не является частным случаем операции (5).

В силу предложения 1 , в рассматриваемой ситуащии стохастическая кубическая матрица должна являться прямой суммой квадратных стохастических матриц. Поэтому кубическая матрица $\left\|p_{i, j, k}\right\|$ является стохастической, если $\sum_{k} p_{i, j, k}=1$ для всех $i, j \in \Gamma$. Легко проверяется полугрупповое свойство совокупности таких стохастических матриц. В самом деле, если $\left\|p_{i, j, k}\right\|$ и $\left\|q_{r, s, t}\right\|$ стохастичны в определенном сейчас смысле, то $(i, j, r)$-й элемент $c_{i, j, r}$ их произведения в смысле (2) равен $c_{i, j, r}=\sum_{k} p_{i, j, k} q_{k, j, r}$, и поэтому

$$
\sum_{r} c_{i, j, r}=\sum_{k} p_{i, j, k} \sum_{r} q_{k, j, r}=1
$$

3. Вероятностные интерпретации. Здесь мы рассмотрим лишь некоторые из возможных вероятностных интерпретаций стохасти- 
ческих кубических матриц с умножением (2)--(5).

а) Умножение (2) интерпретируется тривиально. Элемент $p_{i, j, k}$ стохастической матрицы $P=\left\|p_{i, j, k}\right\|$ задает вероятность перехода частицы типа $j$ из состояния $i$ в состояние $k$. Если $P_{j}$ обозначает $j$-й слой матрицы $P$, то, согласно определению умножения $(2), j$-й слой матрицы $P^{n}$ равен $P_{j}^{n}$. ПІоэтому матрице $P$ можно сопоставить прямую сумму независимых марковских блужданий на $\Gamma$ с матрицами перехода $P_{1}, P_{2}, \ldots, P_{s}$. Понятно, что это блуждание можно рассматривать и как блуждание одной частицы ка состояниях $\Gamma^{s}$ с матрицей перехода, являюпейся кронекеровским произведением матриц $P_{j}, j \in \Gamma$.

б) Обратимся к вероятностной интерпретации умножения (3). Для стохастической матрицы $P=\left\|p_{i, j, k}\right\|$ типа $(2,3), \sum_{j, k} p_{i, j, k}=1$, величину $p_{i, j, k}$ можно интерпретировать как вероятность появления за единицу времени частицы типа $k$ в результате взаимодействия частиц типов $i$ и $j$. Тогда элемент $p_{i, j, r}^{(n)}$, стоящий на $(i, j, r)$-м месте матрицы $P^{n}$, можно трактовать как вероятность появления частицы типа $r$ через $n$ единиц времени из частищы типа $i$ при условик, что на первом шаге она взаимодействует с частищей типа $j$.

в) Так как для стохастической матрицы $P=\left\|p_{i, j, k}\right\|$ типа $(1,2)$ $\sum_{i, j} p_{i, j, k}=1$, то $p_{i, j, k}$ можно интерпретировать как вероятность для частицы типа $k$ иметь упорядоченную родительскую пару типов $i$ и $j$. Тогда элемент $p_{i, j, r}^{(n)}$, стоящий на $(i, j, r)$-м месте матрицы $P^{n}$ (с умножением (3)), можно трактовать как вероятность для частицы типа $r$, полученной через $n$ единиц времени, иметь исходную родительскую упорядоченную пару частиц типов $i$ и $j$.

г) Рассмотрим матрицу $P=\left\|p_{i, j, k}\right\|$, дважды стохастическую, скажем, относительно последнего индекса, с операцией умножения (3). Поскольку для такой матрицы $\sum_{k} p_{i, j, k}=1 / s$, то можно считать, что за единицу времени с вероятностью $1 / s$ появится частица типа $j$ и за то же время с вероятностью $s p_{i, j, k}$ появится частица типа $i$, которая, взаимодействуя с появившейся частицей типа $j$, производит частипу типа $k$. Элемент $p_{i, j, r}^{(n)}$, стоящий на $(i, j, r)$-м месте матрицы $P^{n}$, можно рассматривать как вероятность появления частицы типа $r$ из частицы типа $i$ за $n$ единиц времени при условии, что на первом шаге появляется частица типа $j$.

Заметим, что величину $\sum_{m}(1 / s) s p_{k, m, r}=p_{k, r}$ можно трактовать как вероятность перехода за один шаг частицы типа $k$ в частицу типа $r$ при взаимодействии с любой частицей типа $m$, ибо $\sum_{r} p_{k, r}=1$. Следовательно, описанный процесс можно представить следуюшим образом. На начальном этапе с вероятностью $1 / s$ выбирается частица любого из $s$ типов, скажем, типа $j$, дающая при взаимодействии с частицей типа $i$ частицу типа $k$. Дальнейшее появление частип происходит по схеме марковской цепи с матрицей перехода $\left\|p_{k, r}\right\|$.

п) Можно обобщить процесс, описанщй в п. г), считая, что части-

4 Теория вероятностей и ее применения, № 1 
пы типа $j$ появляются с заданной вероятностью $p_{j}, \sum_{j} p_{j}=1$. Матрицу $\left\|p_{i, j, k}\right\| \mathrm{c}$ неотрицательными элементами, удовлетворяюшими условиям $\sum_{k} p_{i, j, k}=p_{j}, \sum_{j} p_{j}=1$, можно рассматривать как обобшенную дважды стохастическую (по третьему индексу) матрицу. Нетрудно видеть, что такие матрицы образуют полугруппу с общей операцией умножения (5). Аналогично вводится обобщенная дважды стохастичность относительно первого и второго индексов.

Интерпретация умножения (4) вполне аналогична описанным, с той лишь разницей, что появление частицы типа $j$ происходит не на первом, а на последнем этапе.

ж) Пусть $P=\left\|p_{i, j, k}\right\|$ и $Q=\left\|q_{k, m, r}\right\|$ - две стохастические матрицы типа $(2,3)$ и умножение определяется $(5)$. Тогда $(i, d, r)$-й элемент $c_{i, d, r}$ произведения $P Q$ равен $c_{i, d, r}=\sum_{j \circ m=d} \sum_{k} p_{i, j, k} q_{k, m, r}$.

Если рассматривать $p_{i, j, k}$ как вероятность превращения за единицу времени частицы типа $i$ в частицу типа $k$ под воздействием фактора $j$ и считать, что суперпозиция действуюших факторов есть снова фактор, определяемый операпией о, то $c_{i, d, r}$ задает вероятность преврашения частицы типа $i$ в частицу типа $r$ за два временны́х такта под действием суперпозиции любых двух факторов $j$ и $m$, для которых $j \circ m=d$. Аналогичную трактовку получают и элементы любой степени $P^{n}$.

Заметим, что ассоциативность умножения о обеспечивает однозначную определенность фактора за $n$ единиц времени по факторам, действующим на каждом шаге. Кроме того, ассоциативность гарантирует сохранение рекуррентных соотношений для вероятностей перехода, как и в обычных пепях Маркова.

4. Процесс марковского взаимодействия. Процесс, название которого вынесено в заголовок, был в общих словах определен во введении. Определяемый процесс фактически не связан с умножением кубических матриц, а использует лишь стохастические матрицы типа $(1,2)$ или $(2,3)$. Умножение же $(5)$ потребуется лишь для описания связанных с процессом событий, описываюших его предельное поведение.

Дадим теперь строгое формальное описание процесса.

Процесс марковского взаимодействия (п.м.в.) задается на Г тремя элементами:

а) начальным распределением $\mu=\left\{p_{i}\right\}$ на $\Gamma$;

б) стохастической квадратной матрицей $\left\|p_{i, j}\right\|$ на $\Gamma$;

в) кубической матрицей $\left\|\widetilde{p}_{i, j, k}\right\|$, для которой $\widetilde{p}_{i, j, k} \geqslant 0$ и $\sum_{k} \widetilde{p}_{i, j, k}=1$ при всех $i, j \in \Gamma$.

Кубические матрицы из п. в) будем в дальнейшем называть для краткости вероятностными, а числа $\widetilde{p}_{i, j, k}$ - переходными вероятностяМи.

Прощесс марковского взаимодействия протекает следующим образом. В начальный момент времени с вероятностью $p_{i}$ имеется частица 
типа $i$. За единицу времени с вероятностью $p_{i, j}$ возникает частица типа $j$ и, как результат ее взаимодействия с частицей типа $i$, с вероятностью $\widetilde{p}_{i, j, i_{1}}$ появляется частица типа $i_{1}$. Можно говорить также, щто в результате взаимодействия частица типа $i$ превращается в частицу типа $i_{1}$. В следуюший момент времени с вероятностью $p_{i_{1}, j_{1}}$ появляется частица типа $j_{1}$, при взаимодействии которой с частицей типа $i_{1}$ появляется частица типа $i_{2}$, и т.д.

Траекторию п.м.в. можно описать двустрочной матрицей

$$
\left(\begin{array}{llll}
i, & i_{1}, & i_{2}, & \ldots \\
j, & j_{1}, & j_{2}, & \ldots
\end{array}\right)
$$

где в первой строке указаны типы эволющионирующих частиц в последовательные моменты времени, а во второй - типы частиц, появившиеся для взаимодействия. Эти последние можно трактовать либо как маркировку самой частицы типа $i_{k}$, либо как маркировку перехода частицы типа $i_{k}$ в частицу типа $j_{k}$. Ясно, что вероятность перехода $i$ в $i_{1}$ при взаимодействии $i$ с $j$ равна

$$
p_{i, j, i_{1}}=p_{i, j} \widetilde{p}_{i, j, i_{1}}
$$

и матрища $\left\|p_{i, j, i_{1}}\right\|$ является стохастической матрицей типа $(2,3)$. Ясно также, что произведение $p_{i, j, i_{1}} p_{i_{1}, j_{1}, i_{2}} \cdots p_{i_{n-1}, j_{n-1}, i_{n}}$ задает вероятность траектории $\left(\begin{array}{ccccc}i, & i_{1} & \ldots, & i_{n-1}, & i_{n} \\ j, & j_{1} & \ldots, & j_{n-1}, & .\end{array}\right)$.

Предложение 8. Суиеств ует взаимооднозначное соответствие между матричами переходных вероятностей п.м.в. и кубическими стохастическими матричами типа $(2,3)$.

Д о к а з а те л ь с т в. Как уже отмечалось, матрица с элементами (7) является стохастической типа $(2,3)$. Наоборот, если задана стохастическая матрица $\left\|p_{i, j, i_{1}}\right\|$ типа $(2,3)$, то, положив $p_{i, j}=\sum_{i_{1}} p_{i_{, j}, i_{1}}$, получим, очевидно, стохастическую квадратную матрицу $\left\|p_{i, j}\right\|$. Далее, положив $\widetilde{p}_{i, j, i_{1}}=p_{i, j, i_{1}} / p_{i, j}$, получаем вероятностную матрицу $\left\|\widetilde{p}_{i, j, i_{1}}\right\|$, что также очевидно. Предложение доказано.

Обычная марковская щепь, конечно, является частным случаем п.м.в., когда $\widetilde{p}_{i, j, i_{1}}=\widetilde{p}_{i_{i, i_{1}}}$ при всех $j \in \Gamma$. Однако, в отличие от марковской цепи, предельное поведение вероятностей иметь частицу типа $i$ на $n$-м шаге существенно зависит от множества частиц $j, j_{1}, \ldots, j_{n-1}$, обеспечивающих взаимодействие. В частности, не совсем ясно, что понимать под эргодичностью п.м.в. Оказывается, что умножение (5) кубических матриц представляет возможность ответить на эти вопросы. Но сначала докажем

Предложение 9. Пусть $P=\left\|p_{i, j, k}\right\|-$ стохастическая матрица типа $(2,3)$, определяющая, согласно предложению 8 , п.м.в. Элемент $p_{i, d, k}^{(n)}$ матрицы $P^{n}=\left\|p_{i, d, k}^{(n)}\right\|$ с умножением (5) задает вероятность перехода в рассматриваемом п.м.в. частицы типа $i$ за п единич времени 
6 частицу типа $k$ при произвольном наборе $j, j_{1}, \ldots, j_{n-1}$, удовлетворяющем условию $j \circ j_{1} \circ \cdots \circ j_{n-1}=d$.

Д о к а з а т е л ь с т в о. По определению умножения (5),

$$
p_{i, d, k}^{(n)}=\sum_{j \circ j_{1} \circ \cdots \circ j_{n-1}=d} \sum_{i_{1}, \ldots, i_{n-1} \in \Gamma} p_{i, j, i_{1}} p_{i_{1}, j_{1}, i_{2}} \cdots p_{i_{n-1}, j_{n-1}, k} .
$$

Заменяя трехиндексные вероятности по формуле (7), получим

$$
\begin{aligned}
p_{i, d, k}^{(n)}= & \sum_{j \circ j_{1} \circ \cdots \circ j_{n-1}=d} \sum_{i_{1}, \ldots, i_{n-1} \in \Gamma} p_{i, j} \widetilde{p}_{i_{, j, i_{1}}} p_{i_{1}, j_{1}} \tilde{p}_{i_{1}, j_{1}, i_{2}} \times \cdots \\
& \times p_{i_{n-1}, j_{n-1}} \tilde{p}_{i_{n-1}, j_{n-1}, k} .
\end{aligned}
$$

Произведение, представляющее обший член этой суммы, равно вероятности перехода частицы типа $i$ в частищу типа $k$ за $n$ единип времени в п.м.в., если частица типа $i$ последовательно производит потомков $i_{1}, i_{2}, \ldots, i_{n-1}, i_{n}=k$ с помощью частиц типов $j, j_{1}, \ldots, j_{n-1}$.

Таким образом, стационарность п.м.в., определяемого матрицей $P$, зависит от выбора умножения, в смысле которого степени $P^{n}$ сходятся.

5. Стационарные кубические матрицы и эргодичность п.м.в. Предельное поведение вероятностей для п.м.в. возможно лишь по отношению к тому или иному множеству последовательностей типов частиц, участвуғщих во взаимодействиях, и тесно связано с понятием стационарности для кубических матриц.

O II р е п е л е н и е 4. Процесс марковского взаимодействия называется эргодическим по отношению к умножению (5), если при этом умножении степени $P^{n}$ отвечающей п.м.в. кубической матрицы $P$ стремятся к матрице II с положительными элементами.

Ясно, что предельная матрица II стационарна, т.е. $P \Pi=\Pi$ и $\Pi^{2}=\Pi$.

Таким образом, сходимость вероятностей перехода частицы типа $i$ в частину типа $k$ с ростом $n$ естественно определяется по отношению к множеству $\left(j, j_{1}, j_{2}, \ldots, j_{n-1}\right)$ типов частищ, появившихся для взаимодействия, для которых $j \circ j_{i} \circ \cdots \circ j_{n-1}=d$.

Произвольная стохастическая матрица $P$ типа $(2,3)$ однозначно представляется в виде

$$
P=\sum_{i, k} \tilde{p}_{i, k} \varepsilon_{i, k}
$$

где $\varepsilon_{i, k}=\sum_{t \in \Gamma} c_{i, t, k}(i, t, k), c_{i, t, k} \geqslant 0, \sum_{t \in \Gamma} c_{i, t, k}=1$, a $\left\|\tilde{p}_{i, k}\right\|-$ compoвождающая стохастическая матрица для $P$. Ясно, что $p_{i, j, k}=\tilde{p}_{i, k} c_{i, j, k}$. Будем называть представление (8) марковским разложением кубической стохастической матрицы $P$.

Полугрупповая операция о определяет на $\Gamma$ полугруппу $G$. Ниже, если нужно подчеркнуть полугрупповую структуру $\Gamma$, мы будем писать $G$ вместо $\Gamma$. 
Обозначим $M^{+}(G)$ совокупность всевозможных линейных комбинаций элементов $G$ с неотрицательными коэффициентами. Операция о естественно индуцирует на $M^{+}(G)$ полугрупповую операцию. Полугруппа $M^{+}(G)$ включает в себя полугруппу $M^{1}(G)$ всех вероятностных мер на $G$.

Определим взаимнооднозначное отображение элементов $\varepsilon_{i, k}=$ $\sum_{t \in \Gamma} c_{i, t, k}(i, t, k)$ в элементы $\widetilde{\varepsilon}_{i, k}=\sum_{t \in G} c_{i, t, k} t \in M^{1}(G)$.

Предложение 10. Справедливо равенство

$$
\left(\varepsilon_{i, k} \circ \varepsilon_{k, m} \tilde{)}=\widetilde{\varepsilon}_{i, k} \circ \widetilde{\varepsilon}_{k, m} .\right.
$$

Д о к а з а т е л ь с т в о. По определению умножения (5),

$$
\begin{aligned}
\varepsilon_{i, k} \circ \varepsilon_{k, m} & =\left(\sum_{u \in \Gamma} c_{i, u, k}(i, u, k)\right) \circ\left(\sum_{v \in \Gamma} c_{k, v, m}(k, v, m)\right) \\
& =\sum_{u, v \in \Gamma} c_{i, u, k} c_{k, v, m}(i, u \circ v, m) \\
& =\sum_{t \in \Gamma}\left(\sum_{u \circ v=t} c_{i, u, k} c_{k, v, m}\right)(i, t, m) .
\end{aligned}
$$

Поэтому

$$
\begin{aligned}
\left(\varepsilon_{i, k} \circ \varepsilon_{k, m}\right) & =\sum_{t \in G}\left(\sum_{u \circ v=v} c_{i, u, k} c_{k, v, m}\right) t \\
& =\left(\sum_{u \in G} c_{i, u, k} u\right) \circ\left(\sum_{v \in G} c_{k, v, m} v\right)=\widetilde{\varepsilon}_{i, k} \circ \widetilde{\varepsilon}_{k, m} .
\end{aligned}
$$

Предложение доказано.

В силу (8) отображение естественным образом линейно и гомоморфно продолжается на полугруппу стохастических кубических матриц типа $(2,3)$. Это приводит к необходимости рассмотрения квадратных матриц с элементами из $M^{+}(G)$ с обычным умножением.

О п р е д е л е н и е 5. Квадратная матрица $\left\|\delta_{i, j}\right\|, \delta_{i, j} \in M^{+}(G)$ называется стохастической матрицей над $M^{+}(G)$, если $\sum_{k \in \Gamma} \delta_{i, k} \in$ $M^{1}(G)$ при всех $i \in \Gamma$.

Легко убедиться, что такие матрицы образуют выпуклую полугруппу. Для дальнейшего принципиальным является

Предложение 11. Полугруппа кубических стохастических матрич типа $(2,3)$ с умножением (5) изоморфна полугруппе стохастических матрич над $M^{+}(G)$.

Д ок а 3 а т е л ь т в о. Пусть $P=\sum_{i, k \in \Gamma} p_{i, k} \varepsilon_{i, k}-$ марковское разложение стохастической кубической матрицы $P$ типа $(2,3)$. Понятно, что соответствие $\psi: P \longrightarrow\left\|\widetilde{p}_{i, k} \widetilde{\varepsilon}_{i, k}\right\|$ является взаимнооднозначным и выпуклым, а в силу предложения 10 и гомоморфным. Кроме того, ясно, 
что матрица $\left\|\tilde{p}_{i, k} \varepsilon_{i, k}\right\|$ стохастична в смысле определения 5 . Предложение доказано.

Доказанное предложение дает возможность, по крайней мере, для умножений (3) и (4), сразу описать стационарные матрицы.

Действительно, для распределений $\mu_{1}$ и $\mu_{2}$ с умножением (3) справедливо равенство $\mu_{1} \circ \mu_{2}=\mu_{1}$, а для умножения (4) - равенство $\mu_{1} \circ \mu_{2}=$ $\mu_{2}$. Поэтому как для умножения (3), так и для умножения (4) имеем: $\mu \circ \mu=\mu^{\circ 2}=\mu$.

Предложение 12. Для общей кубической стационарной эргодической матрицы $P$ с умножением (3) соответствие $\psi$ имеет вид $\Pi$ І = $\left\|\pi_{i, k}\right\|$, гde $\pi_{i, k}=p_{k} \varepsilon_{i}, p_{k} \geqslant 0, \sum_{k} p_{k}=1, \varepsilon_{i} \in M^{1}(G)$.

Д о ка за т е ль с т в о. Пусть $\left\|\widetilde{p}_{i, k} \widetilde{\varepsilon}_{i, k}\right\|$ - образ кубической стохастической матрицы $P$ при соответствии $\psi$. Согласно предложению 7 , отображение $P$ в $\left\|\tilde{p}_{i, k}\right\|$ гомоморфно по умножению. Поэтому стационарность $P$ влечет стационарность и эргодичность $\left\|\widetilde{p}_{i, k}\right\|$. Поэтому, [2], $\widetilde{p}_{i, k}=p_{k}, \sum_{k \in \Gamma} p_{k}=1$. Следовательно, $\left\|\widetilde{p}_{i, k} \widetilde{\varepsilon}_{i, k}\right\|=\left\|p_{k} \widetilde{\varepsilon}_{i, k}\right\|$. Поскольку в силу стационарности эта матрица идемпотентна, то после возведения ее в квадрат с учетом равенства $\mu_{1} \circ \mu_{2}=\mu_{1}$ в $i$-й строке получим:

$$
p_{1}\left(p_{1} \widetilde{\varepsilon}_{i, 1}+\cdots+p_{s} \widetilde{\varepsilon}_{i, s}\right), \ldots, p_{s}\left(p_{1} \widetilde{\varepsilon}_{i, 1}+\cdots+p_{s} \widetilde{\varepsilon}_{i, s}\right) .
$$

Сравнивая эту строку с $i$-й строкой матрицы $\left\|p_{k} \widetilde{\varepsilon}_{i, k}\right\|$, обнаруживаем, что $\widetilde{\varepsilon}_{i, 1}=\widetilde{\varepsilon}_{i, 2}=\cdots=\widetilde{\varepsilon}_{i, s}=\varepsilon_{i}$ для любого $i \in \Gamma$. Наоборот, очевидно, $\left\|p_{k} \varepsilon_{i}\right\|^{2}=\left\|p_{k} \varepsilon_{i}\right\|$. IІредложение доказано.

Аналогично доказывается

Предложение 13. Для общей кубической стохастической эргодической матрицы $P$ с умножением (4) соответствие $\psi$ имеет вид $\left\|\pi_{i, k}\right\|$, ade $\pi_{i, k}=p_{i} \varepsilon_{i}, p_{i} \geqslant 0, \sum_{i \in \Gamma} p_{i}=1, \varepsilon_{i} \in M^{1}(G)$.

Отметим, что тензорное произведение стационарной квадратной матрицы $\left\|p_{k}\right\|$ и стационарного распределения $\varepsilon$ на $G$, очевидно, является стационарным. В соответствии $\psi$ оно имеет вид $\left\|p_{k} \varepsilon\right\|$, что является частным проявлением предложений 12 и 13.

Рассмотрим теперь частный, но весьма распространенный случай, когда в полугруппе $G$ существует двусторонне инвариантная мера $\varepsilon$, т.е. такая мера, пля которой $\mu \circ \varepsilon=\varepsilon \circ \mu=\varepsilon$ при любом $\mu \in M^{1}(G)$. В нашем случае мера $\varepsilon$ всегда должна иметь носитель, совпадаюший с $G$, так как в противном случае ее носитель должен был бы быть собственным идеалом $G$, наличие которого противоречит свойству однозначной разрешимости операции о.

Для полугрупп с операцией (3) или (4) двусторонне инвариантного распределения не имеется. Так что этот случай принципиально противоположен рассматриваемому. В то же время для произвольной конечной группы $G$ инвариантное распределение на $G$ является единственной двусторонне инвариантной мерой на $G$. 
В дальнейшем стохастические матрицы над $M^{1}(G)$ нам будет удобно представлять в виде $\left\|p_{i j} \Delta_{i j}\right\|$, где $\Delta_{i j}$ - вероятностные распределения на $G$, а $\left\|p_{i j}\right\|$ - стохастическая матрица. Для удобства положим $\left\|p_{i j} \Delta_{i j}\right\|=(P, \Delta)$, где $P=\left\|p_{i j}\right\|, \Delta=\left\|\Delta_{i j}\right\|$. В частности, пусть $\varepsilon=\|\varepsilon\|$ - матрица, в которой каждый элемент есть двусторонне инвариантная мера $\varepsilon, T=\left\|p_{k}\right\|$ - стационарная стохастическая квадратная матрица. Тогда имеет место

Лемма 2. Для любых көадратных стохастических матрий $P=$ $\left\|p_{i j}\right\|, Q=\left\|q_{j m}\right\| u \Delta=\left\|\Delta_{i j}\right\|, \Delta_{i j} \in M^{1}(G)$, справедливы равенства

$$
(P, \Delta)(Q, \varepsilon)=(P Q, \varepsilon), \quad(Q, \varepsilon)(P, \varepsilon)=(Q P, \varepsilon) .
$$

Док а зате ль с т в о. Действительно, так как мера $\varepsilon$ двусторонне инвариантна, то $\Delta_{i j} \varepsilon=\varepsilon$ и $\varepsilon \Delta_{i j}=\varepsilon$. Поэтому непосредственное вычисление дает

$$
\left\|p_{i j} \Delta_{i j}\right\| \cdot\left\|q_{j m} \varepsilon\right\|=\left\|\sum_{j} p_{i j} q_{j m} \Delta_{i j} \varepsilon\right\|=\left\|\left(\sum_{j} p_{i j} q_{j m}\right) \varepsilon\right\|=(P Q, \varepsilon) .
$$

Второе равенство проверяется аналогично. Лемма доказана.

Предложение 14. Пусть задана кубическая стохастическая матрица $P$ типа $(2,3)$. Для сходимости степеней $P^{n} \kappa$ стачионарной с положительньми элементами матриче необходимо и достаточно, чтобы при некотором $n_{0}$ все әлементы $P^{n_{0}}$ были положительными.

Д о к а з а т е л в с т в о. Необходимость очевидна, будем доказывать достаточность. Предположим вначале, что все элементы кубической матрицы $P$ положительны. Тогда ее $\psi$-образ равен $\left\|\tilde{p}_{i j} \Delta_{i j}\right\|$, где $\left\|\widetilde{p}_{i j}\right\|$ - стохастическая сопровождаюшая матрица матрицы $P$. В силу (8) и определения отображения $\psi$ все $\widetilde{p}_{i j}$ больше нуля, а носитель каждого распределения $\Delta_{i j}$ совпадает с $G$. Поэтому при некотором $\delta, 0<\delta<1$, справедливо разложение $\left\|\Delta_{i j}\right\|=\delta\|\varepsilon\|+(1-\delta)\left\|\varepsilon_{i j}\right\|$, $\varepsilon_{i j} \in M^{1}(G)$. Следовательно,

$$
\left\|\tilde{p}_{i j} \Delta_{i j}\right\|=\delta\left\|\tilde{p}_{i j} \varepsilon\right\|+(1-\delta)\left\|\tilde{p}_{i j} \varepsilon_{i j}\right\| .
$$

Возводя обе части (9) в $n$-ю степень и учитывая лемму 2 , получим

$$
\psi\left(P^{n}\right)=\left\|\tilde{p}_{i j} \Delta_{i j}\right\|^{n}=\left\{1-(1-\delta)^{n}\right\}\left\|\tilde{p}_{i j}^{(n)} \varepsilon\right\|+(1-\delta)^{n}\left\|\tilde{p}_{i j} \varepsilon_{i j}\right\|^{n},
$$

где $\left\|\widetilde{p}_{i j}^{(n)}\right\|$ есть $n$-я степень сопровождаюшей матрицы $\widetilde{P}$, а $\left\|\dot{\tilde{p}}_{i j} \varepsilon_{i j}\right\|^{n}$ есть некоторая стохастическая матрица над $M^{+}(G)$. Так как все $\widetilde{p}_{i j}>0$, то $\left\|\widetilde{p}_{i j}^{(n)}\right\|$ сходится к некоторой стохастической стационарной матрице $T$ с положительными элементами. Поэтому $\psi\left(P^{n}\right)$ сходится к матрице $(T, \varepsilon)$. Итак, $P^{n}$ сходится к стохастической матрице, представляющейся тензорным произведением стационарной матрицы $T$ и двусторонне инвариантного распределения $\varepsilon$. 
Если известно, что $P^{n_{0}}$ имеет положительные элементы, то, по доказанному, $\psi\left(P^{n_{0} t}\right)$ сходится к $(T, \varepsilon)$ при $t \rightarrow \infty$. Поэтому

$$
\psi\left(P^{n}\right)=\psi\left(P^{r} P^{n_{0} t}\right)=\psi\left(P^{r}\right) \psi\left(P^{n_{0} t}\right), \quad 0 \leqslant r<n_{0},
$$

при любых $n$. Тогда $\psi\left(P^{r}\right) \psi\left(P^{n_{0}} t\right)=\psi\left(P^{r}\right)\left[(T, \varepsilon)+\delta_{n}\right]$, где матрица $\delta_{n}$ стремится к нулю. Так как $T$ стационарная матрица, то в силу леммы 2 , очевидно, имеем: $\psi\left(P^{r}\right)(T, \varepsilon)=(T, \varepsilon)$. Предложение доказано.

Выше отмечалось, что пролесс марковского взаимодействия, определяясь однозначно кубической стохастической матрицей типа $(2,3)$, вообще говоря, не связан с умножением кубических матриц. Операция о помогает только определить множество частиц $\left\{j, j_{1}, j_{2}, \ldots\right\}$, относительно которого существуют финальные вероятности появления потомков. В зависимости от вида кубической матрицы $P$ может оказаться, что при одной операции о процесс марковского взаимодействия эргодичен, а при другой нет. Поэтому возникает интересный для приложений вопрос, при каких условиях на $P$ можно всегда определить на $\Gamma$ такую операцию, чтобы процесс марковского взаимодействия, определяемый $P$, был эргодичен. Это давало бы стратегию выбора частиц $\left\{j_{m}\right\}$. Предложение 14 дает один такой случай: когда все элементы матрицы $P$ больше нуля, то при любом выборе групповой операции о процесс марковского взаимодействия будет эргодичен. Но тогда возникает естественный вопрос о выборе наипростейшего множества $J_{d}^{(n)}=\left\{j, j_{1}, j_{2}, \ldots, j_{n-1}\right\}$, для которого $j \circ j_{1} \circ \cdots \circ j_{n-1}=d$. Ответ здесь простой. Если Г имеет $s$ элементов, то надо выбирать наипростейшие коммутативные группы из $s$ элементов. Например, рассматривать на Г структуру коммутативной группы сложения по $\bmod s$. Если $s=2^{m}$, то еще лучше рассмотреть прямое произведение $m$ групп второго порядка.

6. Марковские цепи, индуцированные кубическими матрицами. Операции (3), (4) и (5) определяют, подобно квадратным матрицам, преобразование множества $\Gamma$ в себя по правилу $i \circledast(l, j, k)=k$, если $i=l$, и нуль в противном случае, трактуемый как уничтожение. Проверка согласованности операции $\circledast$ с ассоциативностью любого из этих умножений очевидна. На множестве $\Gamma^{2}$ также определяется преобразование его в себя, согласованное с умножением кубических матриц. Для общей операции (5) положим

$$
(h, l) \circledast(k, m, r)= \begin{cases}0, & \text { если } l \neq k, \\ (h \circ m, r), & \text { если } l=k .\end{cases}
$$

Тогда

$$
[(h, k) \circledast(k, m, r)] \circledast(r, s, t)=(h \circ m, r) \circledast(r, s, t)=((h \circ m) \circ s, t) .
$$

С другой стороны,

$$
(h, k) \circledast[(k, m, r)(r, s, t)]=(h, k) \circledast(k, m \circ s, t)=((h \circ(m \circ s), t) .
$$


В силу ассоциативности умножения о получаем:

$$
((h \circ m) \circ s, t)=((h \circ(m \circ s), t) .
$$

В частных случаях операций (3) и (4), соответственно, имеем:

$$
(i, j) \circledast(f, j, k)=(i, k), \quad(i, f) \circledast(f, j, k)=(j, k),
$$

в остальных случаях получаем ноль. Наконец, наряду с этими последними действиями, определяются симметричные к ним:

$$
(i, f) \circledast(i, j, k)=(k, f), \quad(i, f) \circledast(i, j, k)=(k, j) .
$$

Проверка ассоциативности для операции (4) дает:

$$
[(i, f) \circledast(i, j, k)] \circledast(k, m, r)=(k, j) \circledast(k, m, r)=(r, m) .
$$

С другой стороны,

$$
(i, f) \circledast[(i, j, k)(k, m, r)]=(i, f) \circledast(i, m, r)=(r, m) .
$$

Аналогично проверяется согласованность умножения с ассоциативностью операции (3).

Тогда, в соответствии с $(10)$, стохастическая матрица $P$ типа $(2,3)$ естественным образом определяет стохастическую матрицу на множестве $\Gamma^{2}$. Для $(h, l) \in \Gamma^{2}$ положим

$$
(h, l) \circledast P=(h, l) \circledast\left[\sum_{k, m, r} p_{k, m, r}(k, m, r)\right]=\sum_{m, r} p_{l, m, r}(h \circ m, r) .
$$

В данном случае мы предполагаем операцию о оцнозначно разрешимой справа. Поэтому при $m \in \Gamma$ и фиксированном $h$ элемент $h \circ m$ однозначно пробегает все элементы $\Gamma$. С другой стороны, $\sum_{m, r} p_{l, m, r}=1$ при любом $l \in \Gamma$. Итак, на $\Gamma^{2}$ определена дважды стохастическая матрица $\sigma(P)$, элементы которой составлены из элементов $P=\left\|p_{i, j, k}\right\|$. Согласно (13), элемент $P_{(h, l),(d, r)}$ матрицы $\sigma(P)$ равен $p_{l, m_{d}, r}$, где $h \circ m_{d}=d$. Поэтому при любой фиксированной нумерации пар $(h, l)$ для столбцов $c$, составленных из них, будем иметь: $c \circledast P=\sigma(P) c$.

Предложение 15. Отображение $\sigma$ взаимно однозначно, гомомор $\phi$ но и линейно выпукло.

Д ок а з а т е л ь с т в о. Гомоморфизм отображения $\sigma$ следует из согласованности операции $\circledast \mathrm{c}$ ассоциативностью умножения кубических матриц. Действительно, имеем по определению: $c \circledast P Q=\sigma(P Q) c . \mathrm{C}$ другой стороны, в силу согласованности $\circledast$ с ассоциативностью умножения имеем: $c \circledast P Q=\sigma(P) c \circledast Q=\sigma(P) \sigma(Q) c$. Откуда $\sigma(P Q)=$ $\sigma(P) \sigma(Q)$. Взаимная однозначность и линейность отображения $\sigma$ очевидны.

Таким образом, отображение $\sigma$ можно рассматривать как представление алгебры кубических матриц квадратными. Среди частных слу- 
чаев операций о и ® содержится много интересных, но мы их не рассматриваем, так как все они являются интерпретациями предложений 9 и 15 .

В заключение укажем еше один марковский прощесс на $\Gamma^{2}$, естественно порождаемый процессом марковского взаимодействия. Если

$$
\left(\begin{array}{llll}
i, & i_{1}, & i_{2}, & \ldots \\
j, & j_{1}, & j_{2}, & \ldots
\end{array}\right)
$$

- траектория п.м.в., то вероятность перехода пары $\left(i_{m}, j_{m}\right)$ в пару $\left(i_{m+1}, j_{m+1}\right)$, очевидно, равна

$$
P_{i_{m} j_{m}, i_{m+1} j_{m+1}}=\tilde{p}_{i_{m} j_{m}, i_{m+1}} p_{i_{m+1} j_{m+1}} \text {. }
$$

В силу свойств б) и в) определения п.м.в. и соотношения (14), имеем $\sum_{i_{m+1}, j_{m+1}} P_{i_{m} j_{m}, i_{m+1} j_{m+1}}=1$, и, следовательно, вероятности (14) определяют на $\Gamma^{2}$ марковский пропесс. В силу предложения 8 , п.м.в. однозначно определяется кубической матрицей $\left\|p_{i, j, k}\right\|=\left\|p_{i j} \widetilde{p}_{i, j, k}\right\|$, и сопровождаюшая матрища $\left\|\widetilde{p}_{i k}\right\|, \tilde{p}_{i k}=\sum_{j \in \Gamma} p_{i, j, k}$, является стохастической. Ясно, что $\widetilde{p}_{i k}$ есть вероятности перехода частицы $i$ в частицу $k$ независимо от промежуточных взаимодействующих частиц $j$.

Таким образом, возникает обычный марковский процесс, который будем называть сопровождаюшим для п.м.в. Сопровождаюший процесс замечателен тем, что его вероятности перехода за $n$ шагов полностью определяют соответствующие вероятности перехода процесса на $\Gamma^{2}$. Точнее, пусть $\left\|\tilde{p}_{k f}^{(n)}\right\|=\left\|\tilde{p}_{k f}\right\|^{n}$ и $P_{i j, f h}$ есть вероятность перехода за $n$ шагов пары $(i, j)$ в пару $(f, h)$. Тогда нетрудно видеть, что имеет место равенство

$$
P_{i j, f h}^{(n)}=\left(\sum_{t \in \Gamma} \tilde{p}_{i, j, t} \tilde{p}_{t f}^{(n-1)}\right) p_{f h} .
$$

Наконец, необходимо отметить, что поддержка этого направления О. В. Висковым, Э. Л. Пресманом и Ю. В. Прохоровым сильно стимулировала появление этой работы. Кроме того, без большой редакционной помоши О. В. Вискова и его замечаний окончательный текст вряд ли был бы написан. Всем им приношу искреннюю дружескую благодарность.

\section{СПИСОК ЛИТЕРАТУРЫ}

1. Соколов H. $П$. Пространственные матрицы и их приложения. М.: Физматтиз, $1960,300 \mathrm{c}$.

2. IIIряев A. H. Вероятность. М.: Наука, 1980, 575 с. 